\title{
Theoretical and Computational Study of Pitching Airfoils at Different Operating Conditions
}

\author{
\{M. Ossman, M. Y. M. Ahmed*, Amr Elnashar and O. E. Abdel Hamid $\}^{\dagger}$
}

\begin{abstract}
The aerodynamic features of pitching NACA0009 airfoil are explored in the present study. The objective is to investigate the impact of pitching mean angle of attack and amplitude on the temporal variation of airfoil aerodynamic coefficients. Numerical simulation results using a commercial CFD solver are compared with those of the analytical prediction approach. The results of both approaches are found in a good agreement. The evolution of the flowfield structure around the airfoil at the different operating conditions is presented.
\end{abstract}

\section{Introduction}

The aerodynamic features of pitching airfoils have been investigated by many researchers using experimental measurements, theoretical analysis, and numerical simulation approaches. For example, in [1], a variety of experiments on unsteady airfoils and wings were undertaken. Analytical studies on unsteady airfoils were conducted in [2] [3]. The studies [4, 5, 6 and 7] are examples of those adopting simulation approach to understand the aerodynamics of pitching airfoils. Several studies were devoted to compare the three different approaches. Numerical and experimental approaches were compared in [8,9 and 10] whereas numerical and analytical techniques were compared in $[11,12]$.

In [12], Amiralaei studied the influence of oscillation amplitude, reduced frequency, and Reynolds number on the aerodynamic force coefficients and flow pattern for NACA 0012 airfoil oscillation about the zero-mean angle of attack. The amplitude of oscillation varied from $2^{\circ}$ to $10^{\circ}$ whereas the reduced frequencies varied from 0.1 to 0.25 . A numerical simulation was conducted on a two-dimensional model and the results were compared to the theoretical Theodorsen method. Amiralaei confirmed that the amplitude of oscillation and its reduced frequency have a negligible effect on the lift curve slope and the hysteresis loops are only broadened or narrowed depending on the variation of both parameters. The impact of the mean angle of attack was not addressed by Amiralaei. Ghoreyshi and Cummings [11] attempted to reach the limits of theoretical modeling of pitching NACA0012 airfoil at high incidence angles. They compared the Theodorsen solution to a two-dimensional numerical simulation. The cases had reduced frequencies of 0.1 and 1 , amplitudes of $10^{\circ}$ and $15^{\circ}$, and mean incidence angles of $10^{\circ}$ and $14^{\circ}$.

The present work aims to shed more light on the aerodynamic aspects of pitching airfoil and the role of pitching kinematic parameters. This is done via comparing the analytical solution of Theodorsen [14] with a two-dimensional numerical model at different oscillating frequencies. The study also addresses the role of the mean angles of attack.

Mym141101@yahoo.com

Egyptian Armed Forces, Egypt. 


\section{Case Study and Methodology}

\subsection{Case Study}

The case study is NACA0009 airfoil of chord $0.15 \mathrm{~m}$. The freestream conditions refer to flow speed of $51 \mathrm{~m} / \mathrm{s}$ at standard sea level atmospheric conditions. The flow around the airfoil is thus subsonic ( $0.15 \mathrm{Mach}$ ) and fairly turbulent $(\mathrm{Re}=5.23 \mathrm{e} 5$ based on the airfoil chord). The airfoil performs pure pitching about the quarter-chord with frequencies of $6,12.5$, and $25 \mathrm{~Hz}$ corresponding to reduced frequencies of $0.1848,0.385$, and 0.77 , respectively. The amplitude of airfoil oscillation is $6^{\circ}$ whereas the mean angles of attack are $0^{\circ}, 5^{\circ}$ and $10^{\circ}$.

\subsection{Analytical Approach}

The analytical approach is based on the solution of the classical linearized small perturbation potential approach proposed by Theodorsen [14] for simple harmonic oscillation. For an airfoil of chord $2 b$ undergoing a pitching oscillation about a hinge point located at $a b$ from the airfoil mid-chord (where $a$ is the half chord percentage) and frequency $f$, with amplitude of oscillation $\bar{\alpha}$ and incoming velocity U, Fig. 1, the coefficients of airfoil lift and moment (about mid-chord) are expressed as:

$\bar{c}_{l}=\pi k^{2}\left\{-\left[\frac{1}{2}-\frac{i}{k}(1+2 C(k))-\frac{2}{k^{2}} C(k)\right] \bar{\alpha}+\left(\frac{1}{2}+a\right)\left[1-\frac{2 i}{k} C(k)\right] \bar{\alpha}\right\}$

where $C(k)$ is the Theodorsen function of the reduced frequency defined as $k=\frac{\omega b}{2 U}$.

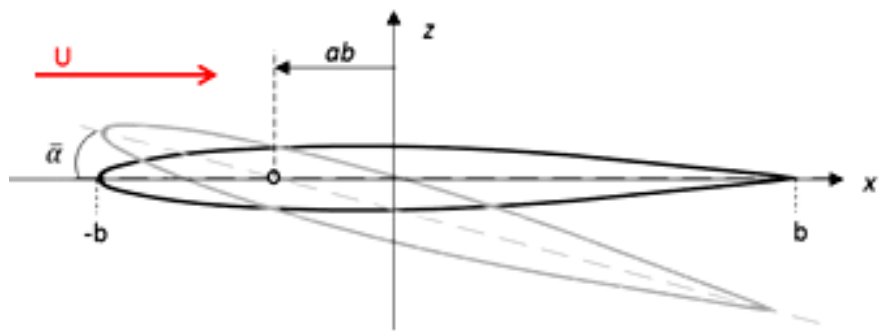

Figure 1 Kinematics of a pitching airfoil

\subsection{Computational Approach}

The domain of interest around the airfoil is extended 30 chord lengths in the upstream and lateral (up and down) directions and 60 chord length in the downstream direction. The computational domain is then split into two zones, the inner and outer zone. The inner zone is movable to perform the prescribed oscillation around a pivot point. The outer zone is fixed and an interface separates the two zones. This technique of domain splitting was successfully adopted in [13], [10]. The boundaries of the computational domain are defined as shown in Fig. 2.

The inner zone mesh is of O-Grid type, and the outer zone mesh is a hybrid grid in four blocks. A structured grid is generated in the two blocks upstream the inner zone center and tri-cells are generated downstream, shown in Fig. 3.

Dynamic (unsteady) simulations are accomplished by continuously oscillating the inner zone according to the prescribed motion using the dynamic mesh technique, while the outer zone is fixed. A grid sensitivity analysis is conducted and validated. The resolution of the grid is controlled by the cell height at the solid wall and number of cells in the direction normal to the wall. The solution was found to converge with $0.5 \%$ variation in the airfoil lift coefficient using a grid with total of 206872 cells, 100 cells normal to the wall in the inner zone, with $0.0001 \mathrm{~m}$ first cell height. 


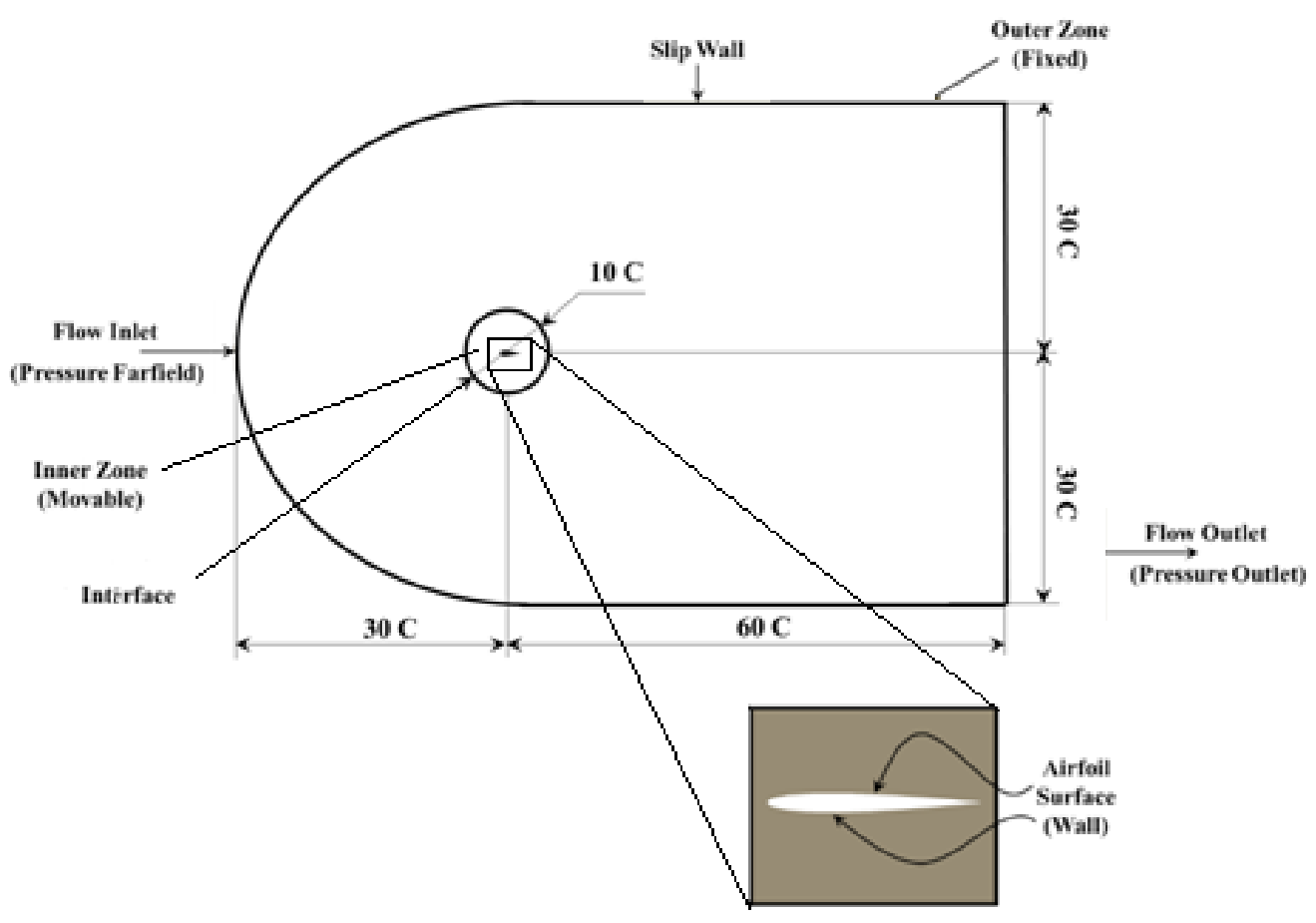

Figure 2 Computational domain definition

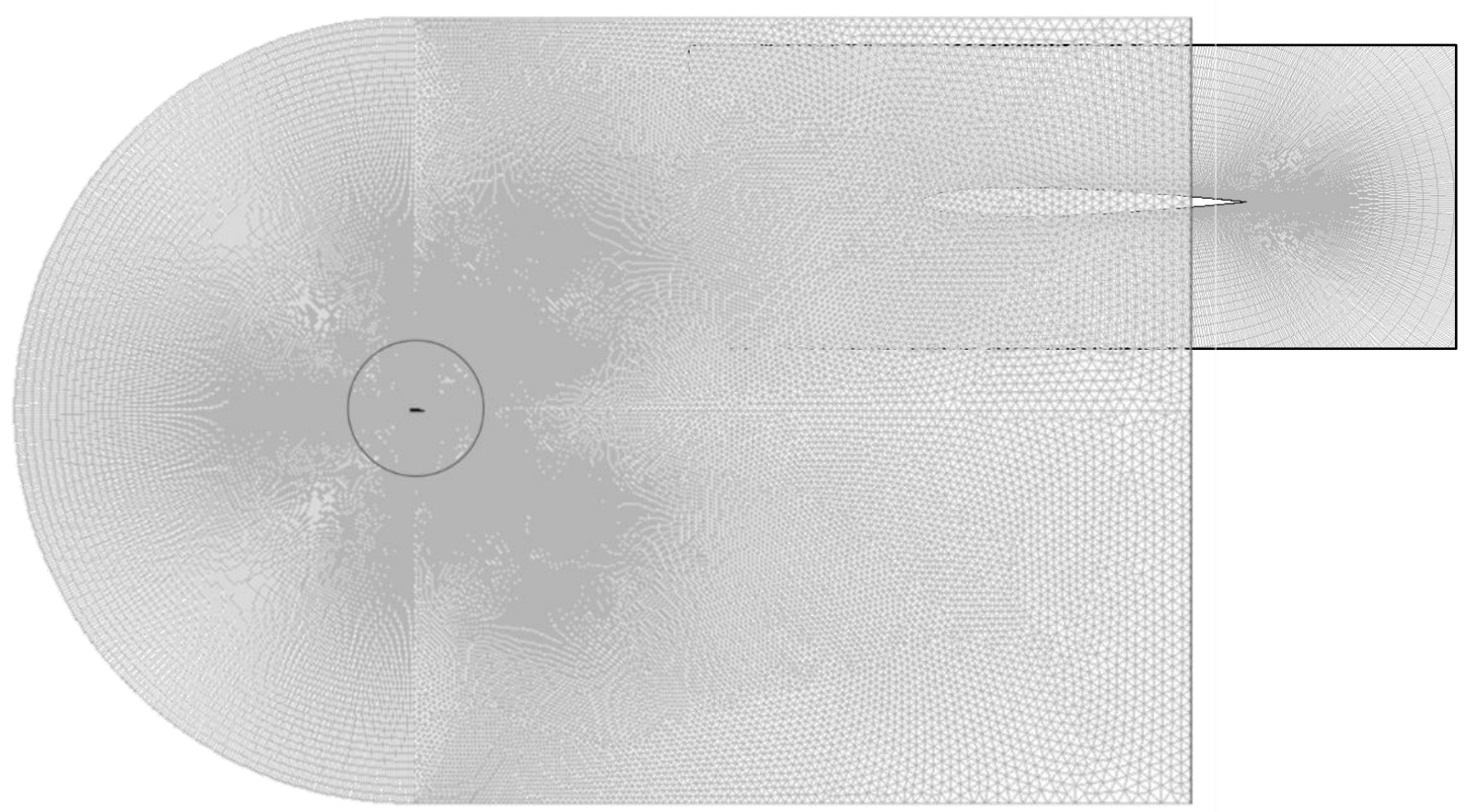

Figure 3 The computational grid with a zoom-in at the airfoil

Various RANS-based turbulence models have been examined by numerically reproducing the measured lift coefficient of a similar case [15]. The Reynolds stress (Linear Pressure) model was found to yield the most accurate results compared with other RANS-based turbulence models. A time step sensitivity analysis is conducted to determine the adequate temporal resolution for the problem in hand. Different time-step sizes have been tested. The temporal variation of airfoil coefficients was found to converge to an invariable pattern using a step size 1/500 of the period. The commercial CFD time-dependent solver used in the study [16] is validated by reproducing the experimental measurements of a pitching airfoil [1]. The used CFD solver is found to yield fairly accurate prediction of the lift coefficient compared with the experimental one. 


\section{Results and Discussion}

\subsection{Impact of Pitching Frequency on the Airfoil Aerodynamic Coefficients}

The impact of pitching frequency on the aerodynamic characteristics of the pitching airfoil is addressed in the present section. The pitching frequencies of $f=6 \mathrm{~Hz}, f=12.5 \mathrm{~Hz}$, and $f=25 \mathrm{~Hz}$ (corresponding to dimensionless frequencies of $0.1848,0.385$, and 0.77 ) are compared for the same mean angle and amplitude, $\alpha_{m}=0^{\circ}$ and $A=6^{\circ}$, respectively. Figure 4a compares the variations of lift coefficient of the airfoil at these three frequencies as calculated by the CFD simulation. The variation of instantaneous incidence angle with time is also plotted in the same figure. Figure $4 \mathrm{~b}$ compares the computational and analytical prediction of the lift coefficient variation at the two frequencies 6 and $25 \mathrm{~Hz}$.

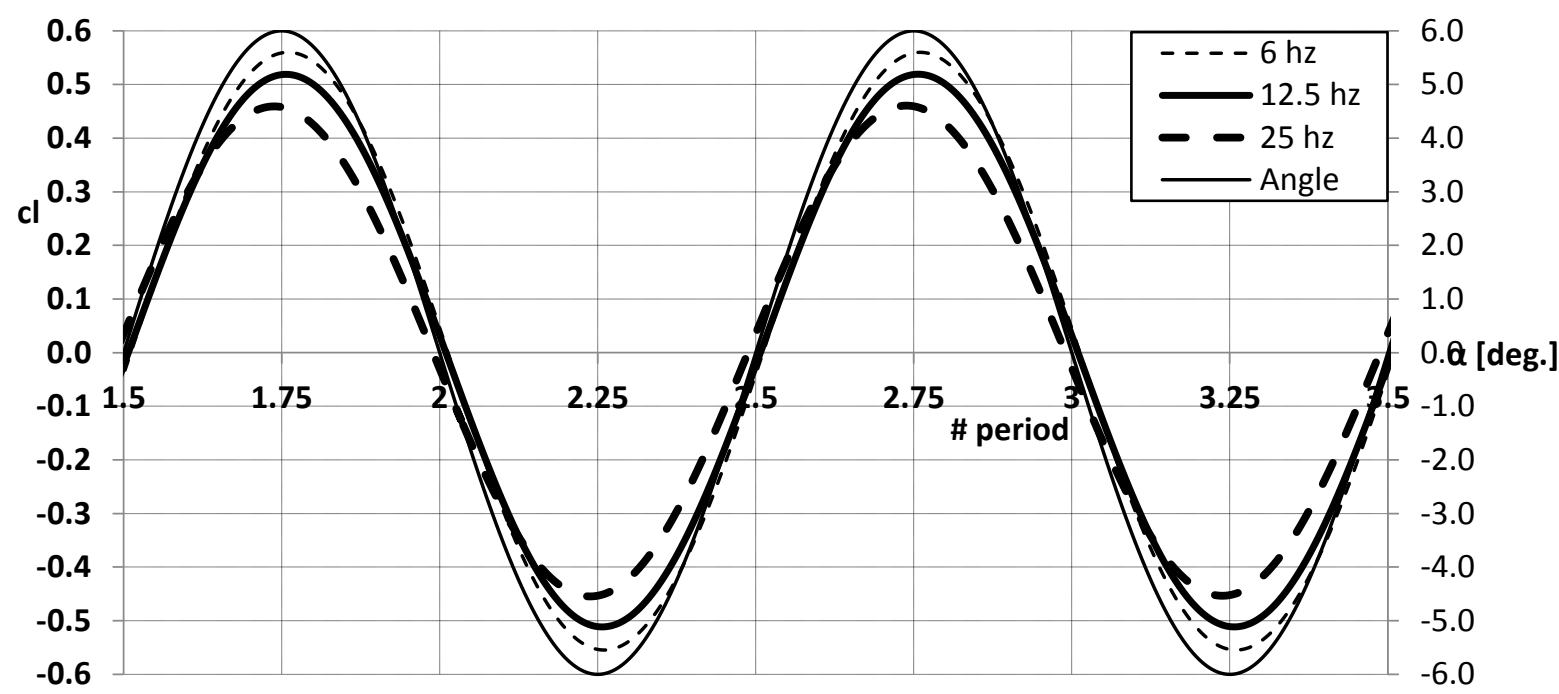

(a)

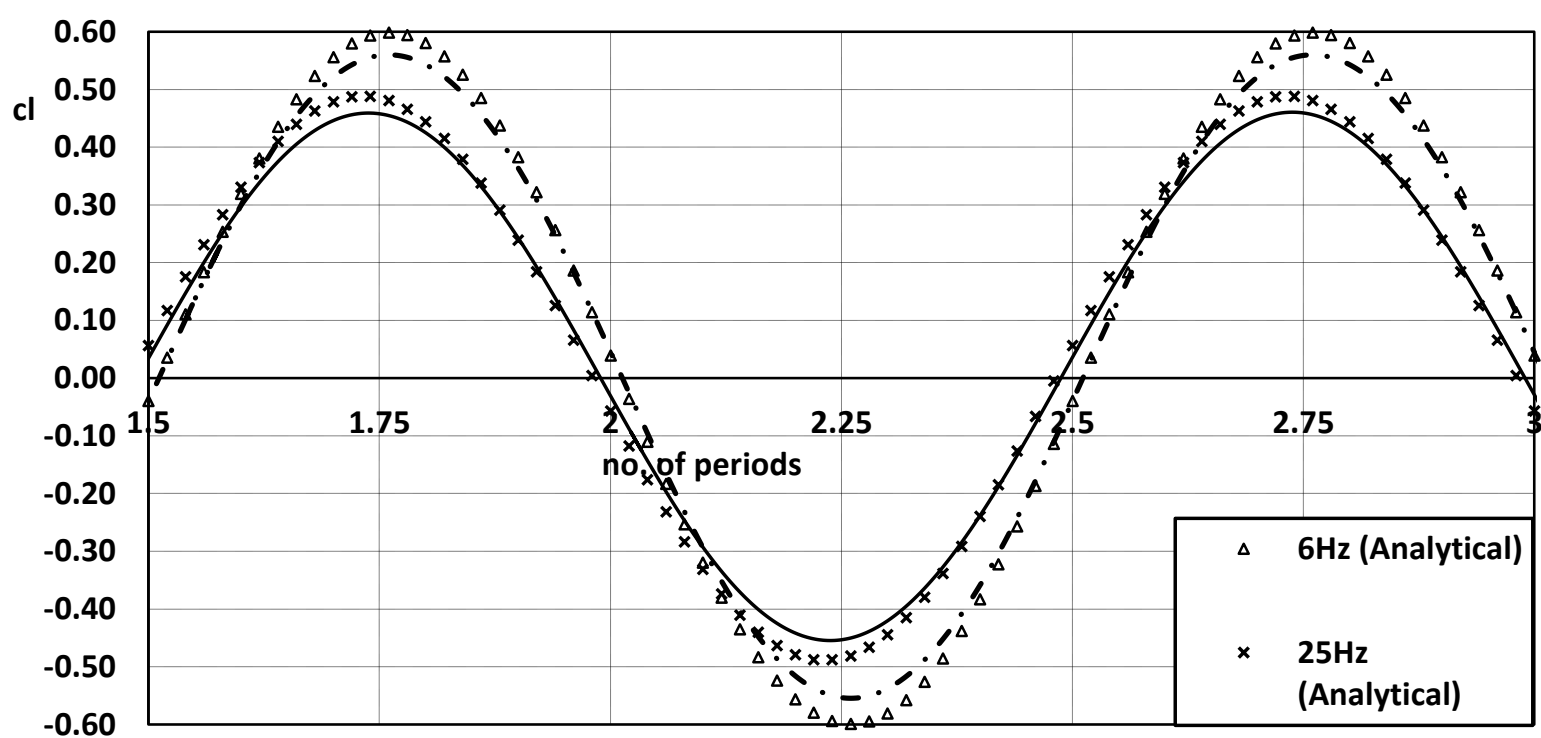

(b)

Figure 4 Impact of pitching frequency on the lift coefficient of the airfoil;

$$
\alpha_{\mathrm{m}}=0^{\circ} \text { and } \mathrm{A}=6^{\circ}
$$


By closely examining the above figures, a set of remarks can be addressed. On the one hand, it is clear that as the pitching frequency increases, the amplitude of lift variation decreases. On the second hand, the analytical and CFD approaches predict the same trend of lift dependence on frequency with no phase shift between the two predictions. Results of the CFD simulations are slightly lower than those of the analytical prediction; the discrepancy reaches a maximum of $6.5 \%$ at the lower and upper peaks at the lowest value of frequency $6 \mathrm{~Hz}$, and decreases as the frequency increase to reach a minimum value of discrepancy of $0.16 \%$ at the higher value of frequency $25 \mathrm{~Hz}$. On the other hand, the phase shift between the airfoil motion and the resulting lift response depends on the motion frequency. At lower frequencies, the lift response lags the airfoil motion whereas at the higher frequency of $25 \mathrm{~Hz}$, the response of the airfoil leads its pitching motion. This phenomenon has been addressed before by [18]. The impact of the pitching frequency on the variation of drag coefficient (based on CFD results) during one complete period is illustrated in Fig. 5 where the hysteresis loops of drag coefficients at the different frequencies are shown.

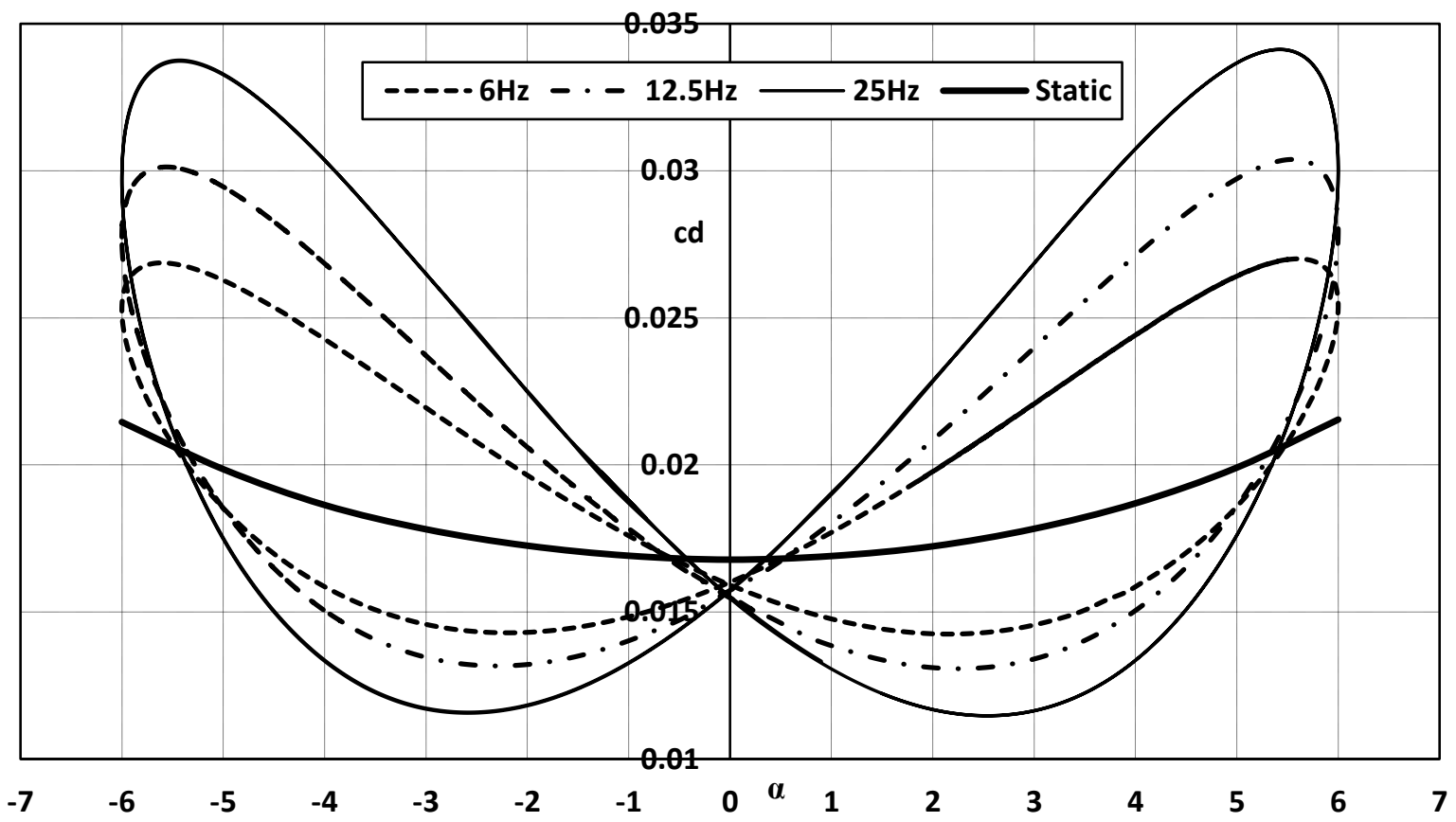

Figure 5 Drag hysteresis loop at different pitching frequencies;

$$
\alpha_{m}=0^{\circ} \text { and } A=6^{\circ}
$$

Increasing the pitching frequency has the role of increasing the amplitude of drag coefficient variation. As the frequency increases, the upper limit of drag increases while its lower limit decreases compared with the stationary airfoil drag. The instantaneous value of drag coefficient at the mean incidence angle $\left(\alpha_{m}=0^{\circ}\right)$ is almost independent of the pitching frequency and remains below that of the stationary airfoil.

\subsection{Impact of Pitching Frequency on the Evolution of Flow Structure During One Complete Period}

Figures 6 and 7 show the evolution of the flowfield structure in terms of the pressure contours and streamlines around the airfoil for $f=6 \mathrm{~Hz}$, and $f=25 \mathrm{~Hz}$, respectively. In both figures, the mean angle of attack and the amplitude of pitching are $0^{\circ}$ and $6^{\circ}$, respectively. The period starts, $t=0$, in the pitch-down direction. 

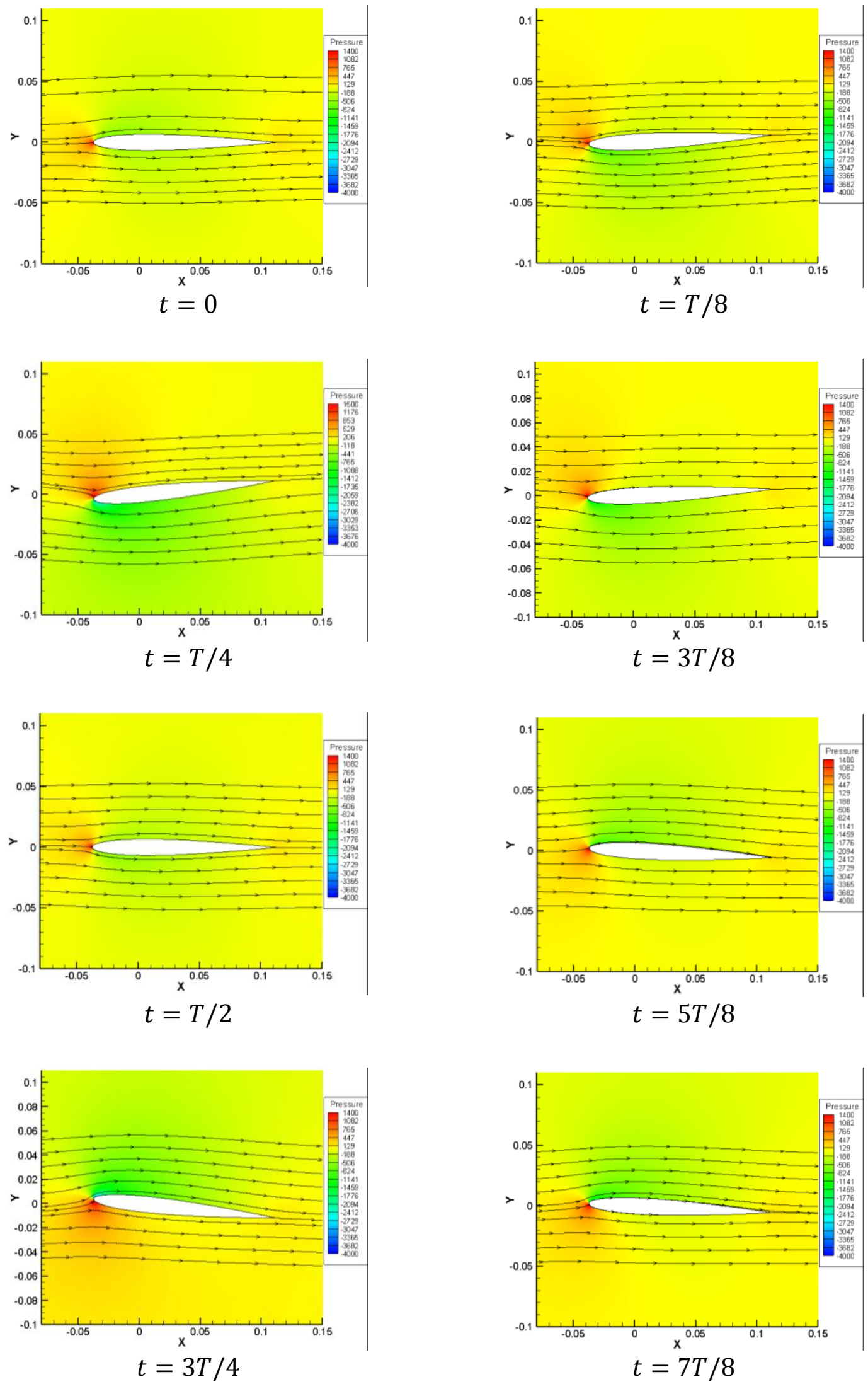

Figure 6 Evolution of the flowfield structure around the airfoil,

$$
\alpha_{m}=0, \alpha_{0}=6, f=6 \mathrm{~Hz}
$$




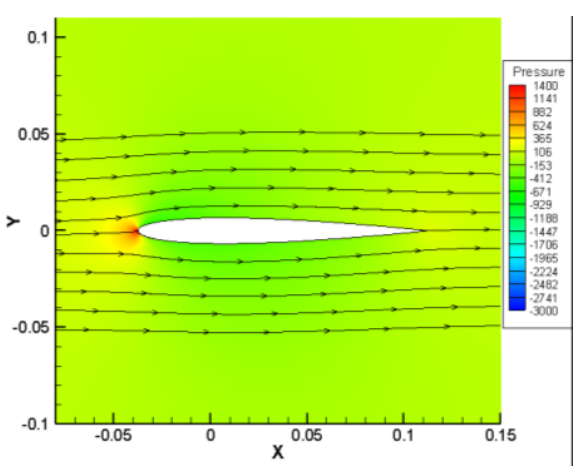

$$
t=0
$$

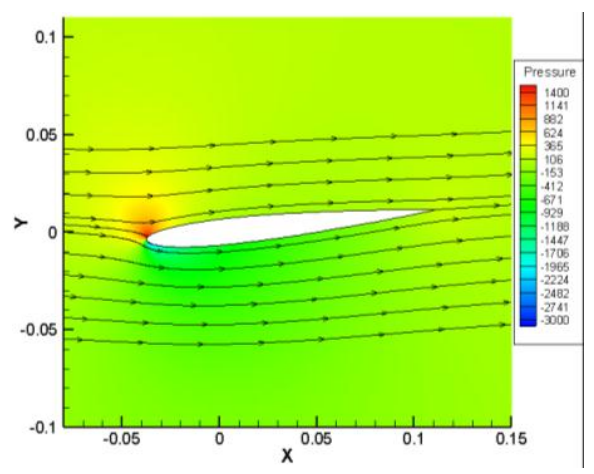

$$
t=T / 4
$$

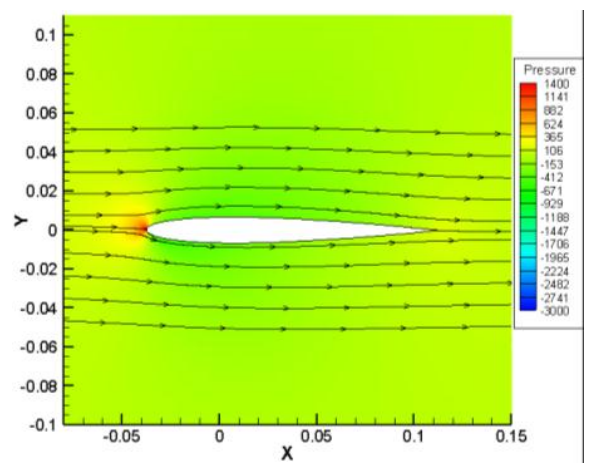

$$
t=T / 2
$$

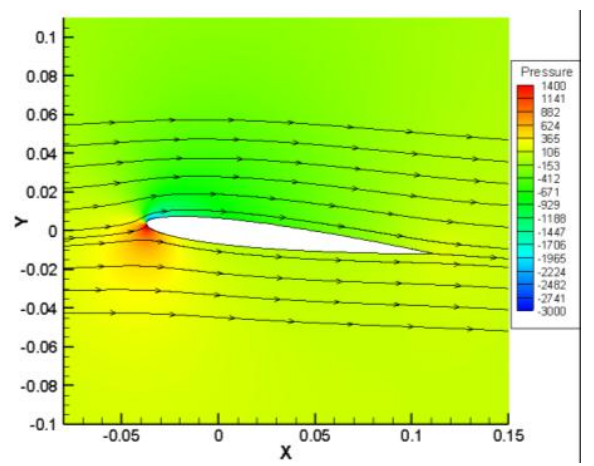

$$
t=3 T / 4
$$

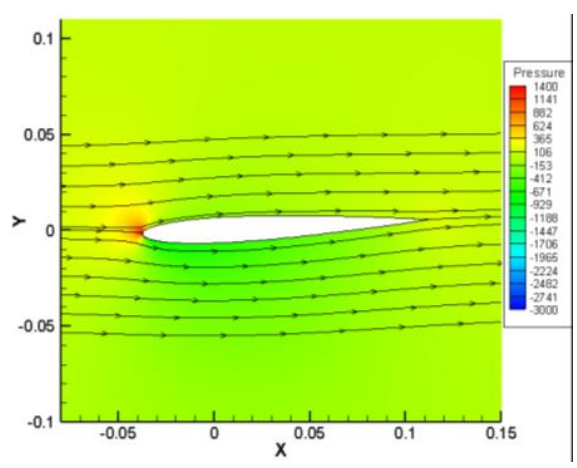

$t=T / 8$

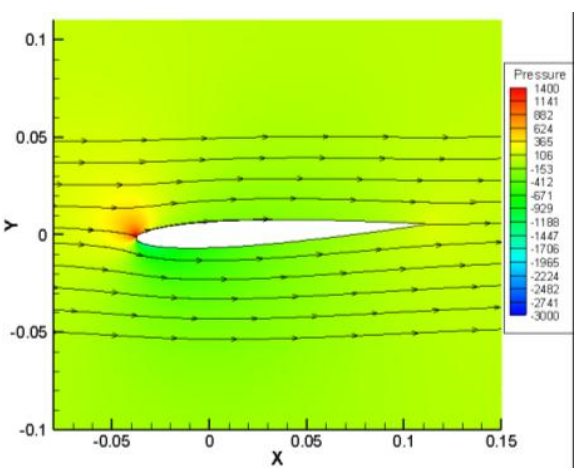

$t=3 T / 8$

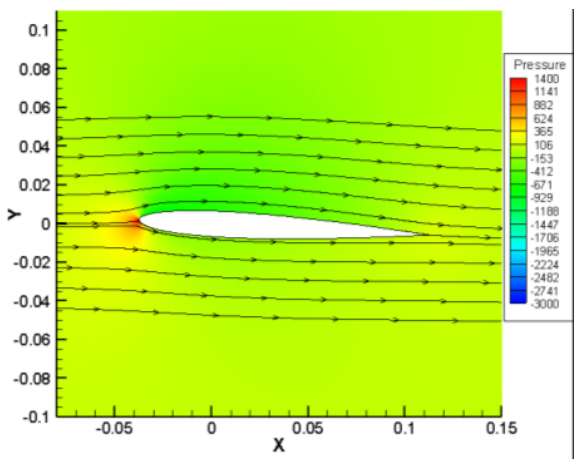

$t=5 T / 8$

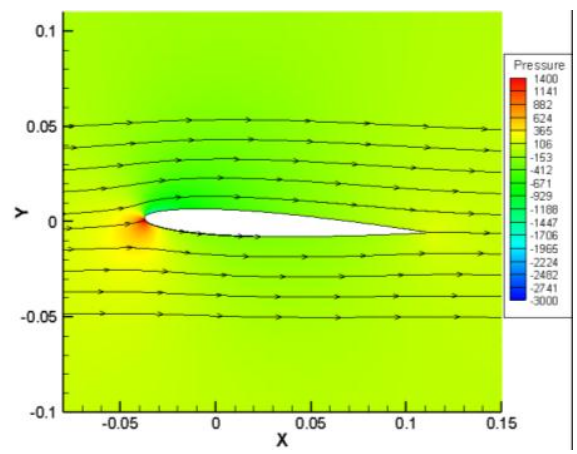

$$
t=7 T / 8
$$

Figure 7 Evolution of the flowfield structure around the airfoil,

$$
\alpha_{m}=0, \alpha_{0}=6, f=25 \mathrm{~Hz}
$$




\subsection{Impact of Mean Angle of Attack on the Airfoil Aerodynamic Coefficients}

Figure 8 shows the lift coefficient variation with time for different mean incidence angles for the same frequency and amplitude of $12.5 \mathrm{~Hz}$ and $6^{\circ}$, respectively. Here, only results from CFD simulations are displayed.

It is clear that, as the mean angle of attack increase, the mean value of lift coefficient increase. At a level where the dynamic stall takes place, as witnessed for the case with MAOA of $10^{\circ}$, a higher values of lift coefficient is achieved in comparison with the static values. As the airfoil approaches its maximum incidence angle $\left(16^{\circ}\right)$, a sudden and sharp lift loss is experienced. This loss is recovered by the vortex shedding and the excited flow around the airfoil restoring a lift regeneration. The impact of the mean angle of attack on the temporal variation of the airfoil drag coefficient is illustrated in Fig. 9. For better illustration, the values for MAOA of $10^{\circ}$ are shown on the secondary vertical axis (to the right).

As shown earlier, for pitching about MAOA of $0^{\circ}$, the drag coefficient shows a symmetric sinusoidal behavior about a mean value with twice the frequency of that of the incidence angle. For pitching about $5^{\circ}$ MAOA, the drag coefficient shows an asymmetric variation about its mean value. A sharp rise in the drag coefficient value is attained for MAOA of $10^{\circ}$; a sharp drop in drag immediately follows. The trend is partially restored afterwards.

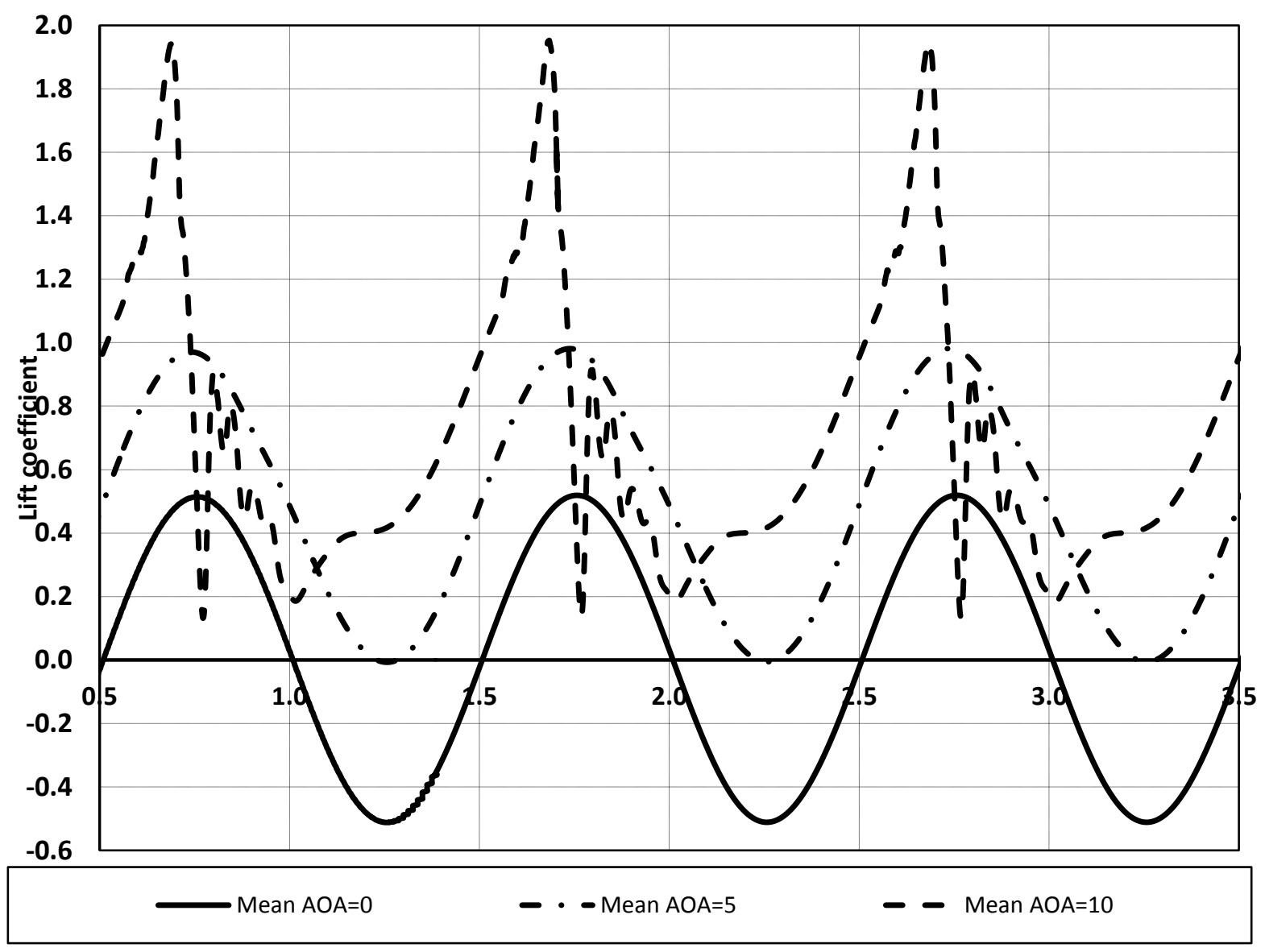

Figure 8 Impact of the mean angle of attack on the temporal variation of the airfoil lift coefficient; $f=12.5 \mathrm{~Hz}$ and $A=6^{\circ}$ 


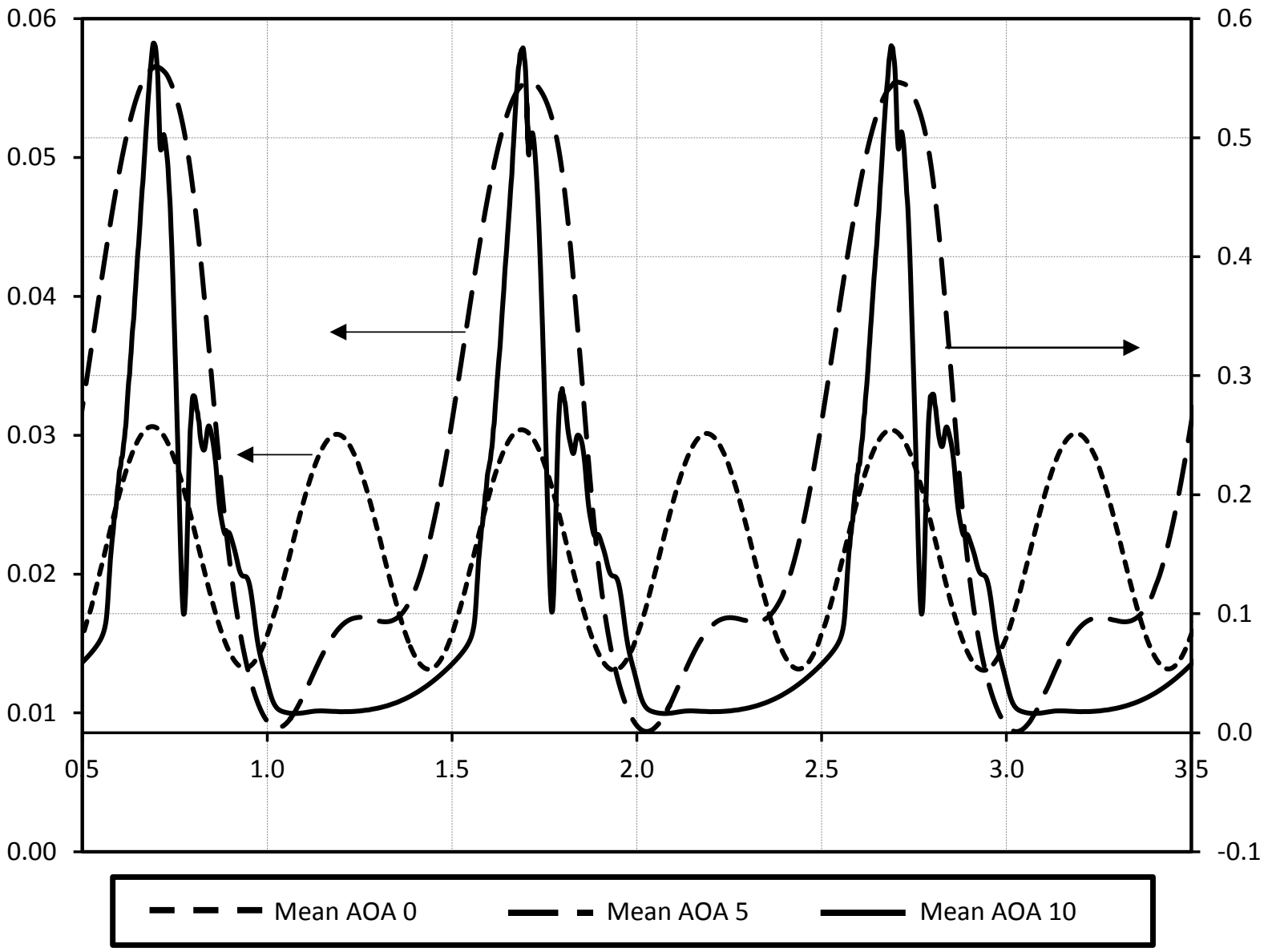

Figure 9 Impact of the mean angle of attack on the temporal variation of the airfoil drag coefficient; $f=12.5 \mathrm{~Hz}$ and $A=6^{\circ}$

\subsection{Impact of Mean Angle of Attack on the Evolution of Flow Structure During One Complete Period}

Figures 10, 11 and 12 address the impact of mean angle of attack on the structure of the flowfield around the airfoil. The period starts, $t=0$, in the pitch-down direction.

\subsection{Lift Components on Pitching Airfoil}

The theoretical approach provides one advantage over CFD simulations that is; the two lift components namely; the circulatory and apparent mass "non-circulatory" terms can be separated thus providing better understanding of physics of lift generation in pitching airfoils. According to Eqn. (1), the airfoil lift coefficient can be written as:

$\bar{c}_{l}=[(1-2 a) \pi i k C(k)+2 \pi C(k)] \bar{\alpha}+\left[a \pi k^{2}+i \pi k\right] \bar{\alpha}$

The coefficient of the first term in the LHS of the above equation is the circulatory term, which links the quasi steady solution to the quasi unsteady one by means of Theodorsen function. The coefficient of the second term is the non-circulatory terms, which is the inertia force generated by the motion of the airfoil, a.k.a., apparent mass terms. The components of lift acting on the airfoil in concern at different frequencies (mean angle and amplitude of $0^{\circ}$ and $6^{\circ}$, respectively) are shown in the figure below. For better illustration, the values of circulatory terms are shown on the main (left) vertical axis whereas the values of non-circulatory terms are shown on the secondary (right) vertical axis. The period starts in the pitch-up direction. 

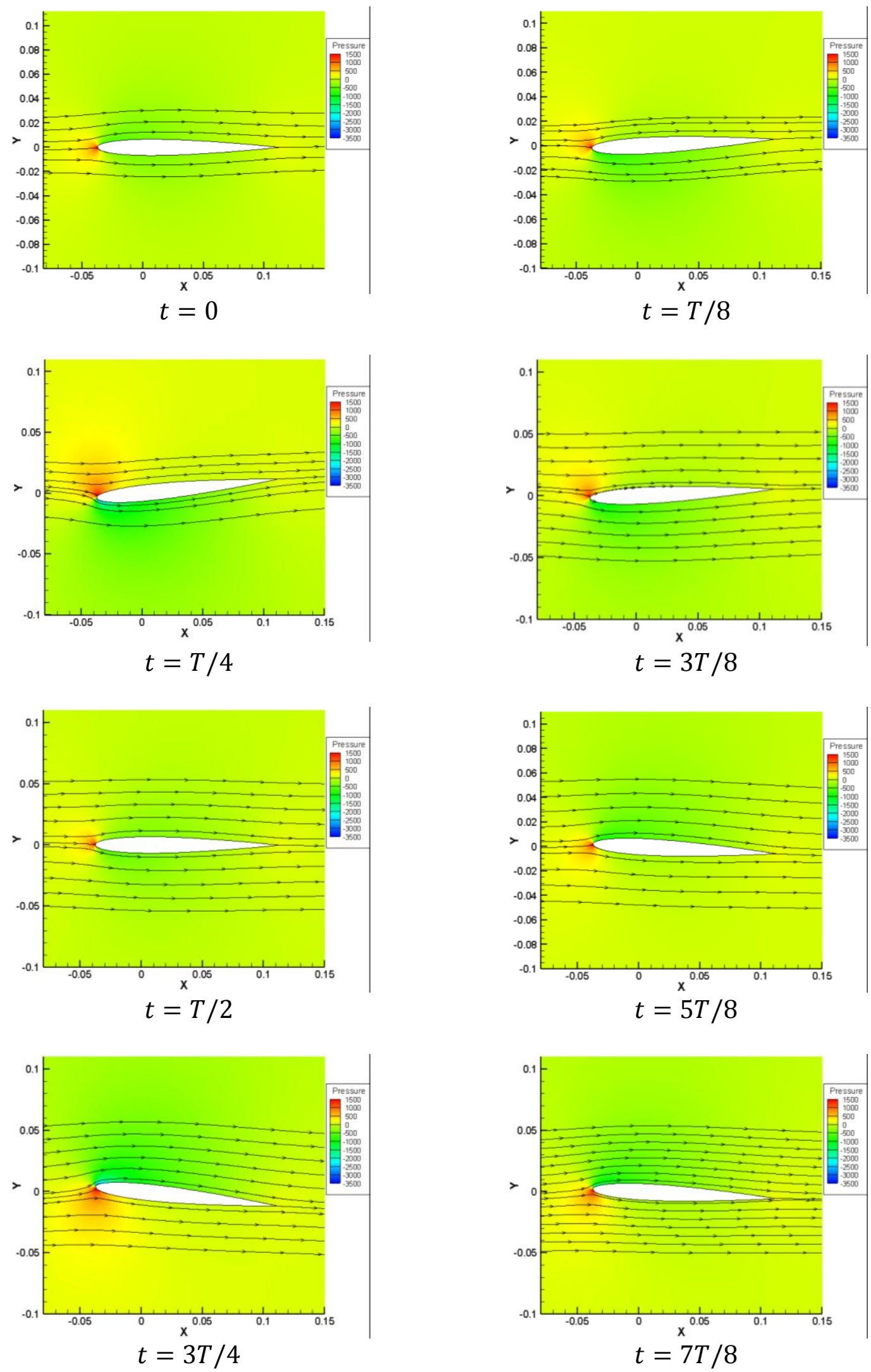

Figure 10 Evolution of the flowfield structure around the airfoil,

$$
\alpha_{\mathrm{m}}=0, \alpha_{0}=6, \mathrm{f}=12.5 \mathrm{~Hz}
$$



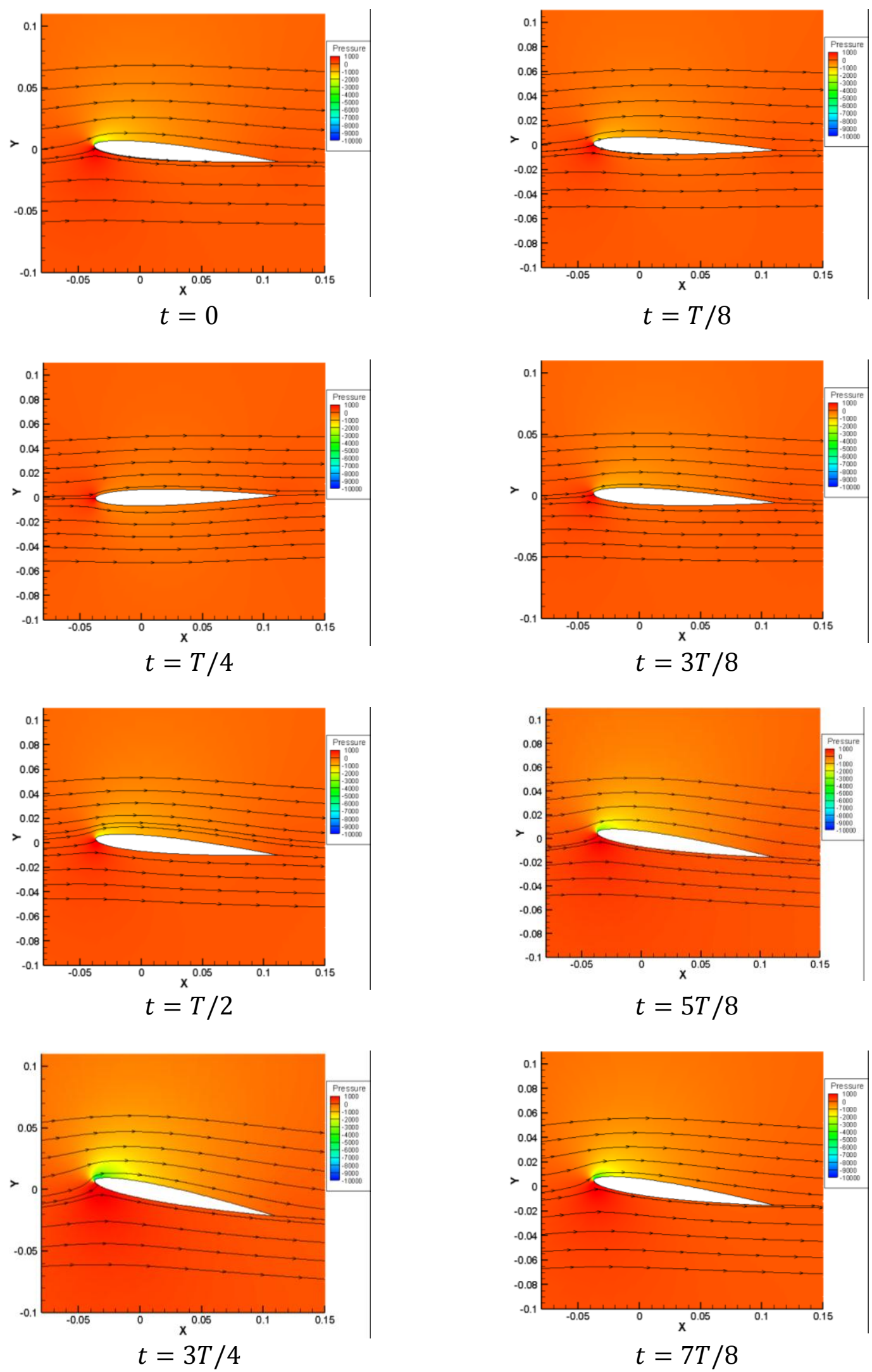

Figure 11 Evolution of the flowfield structure around the airfoil, $\alpha_{\mathrm{m}}=5, \alpha_{0}=6, \mathrm{f}=12.5 \mathrm{~Hz}$ 

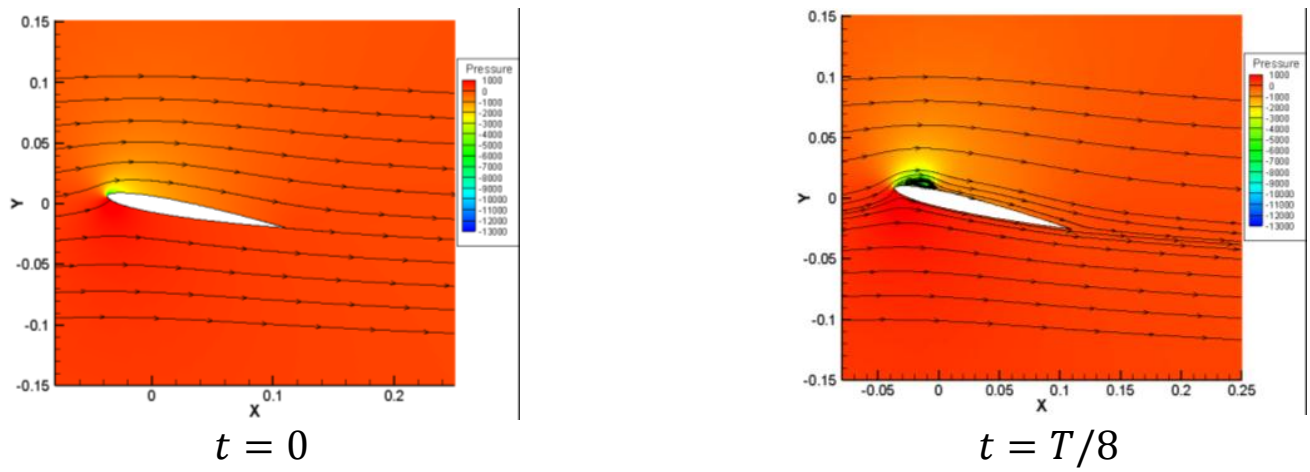

$t=T / 8$
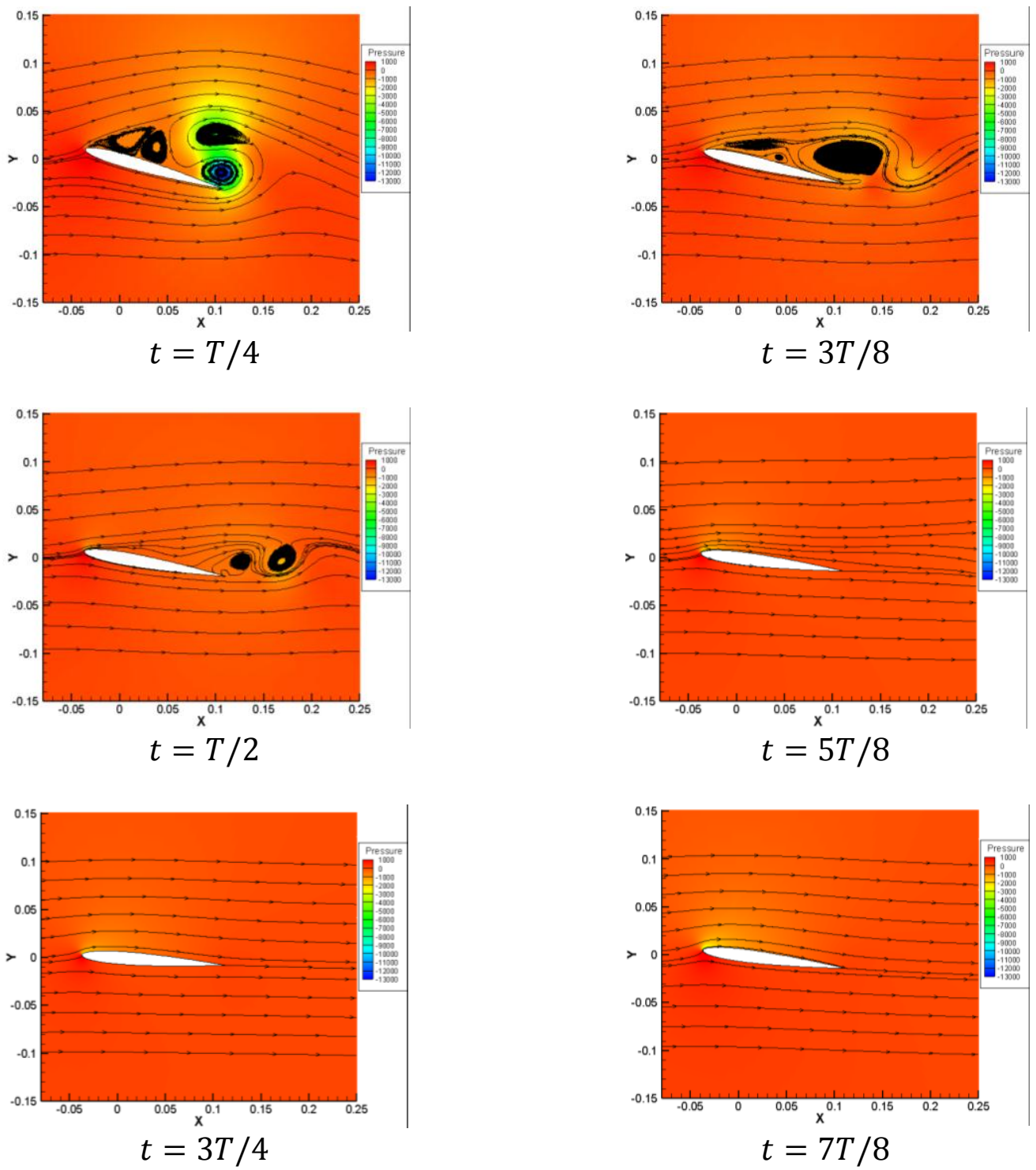

Figure 12 Evolution of the flowfield structure around the airfoil,

$$
\alpha_{\mathrm{m}}=10, \alpha_{0}=6, \mathrm{f}=12.5 \mathrm{~Hz}
$$




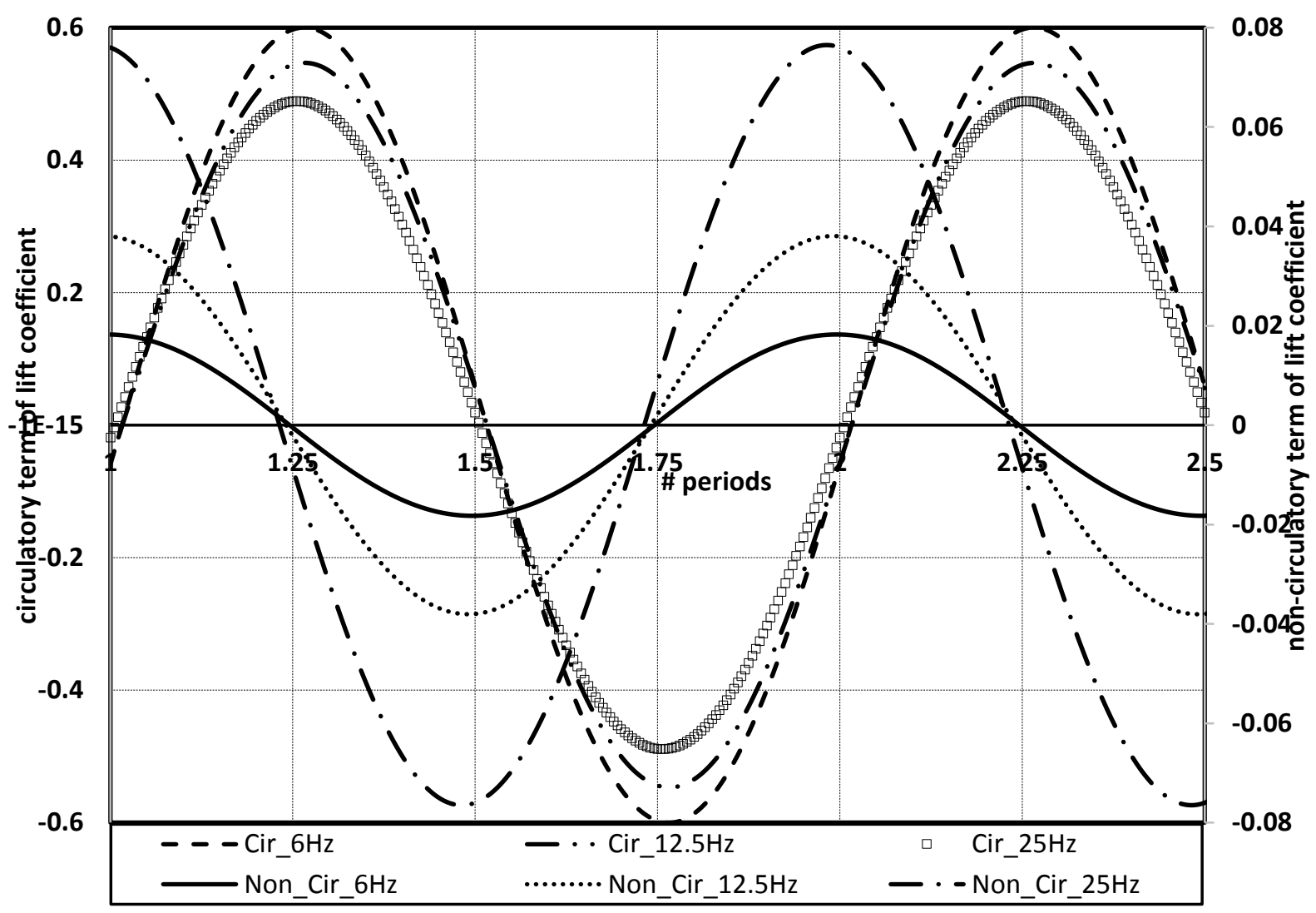

Figure 13 Components of airfoil lift at different pitching frequencies,

$$
\alpha_{\mathrm{m}}=0, \alpha_{0}=6
$$

A number of remarks can be made on the above figure. The circulatory component of lift is only slightly lagging the airfoil motion. In contrast, the non-circulatory component is in a clear phase-difference with the airfoil motion. This may be awed to the nature of generation of each lift component. Since the circulatory term depends on the instantaneous inclination of the airfoil with respect to the freestream, it reaches a maximum at the extremes of the airfoil motion and a minimum near the mean angle of pitching motion. In contrast, the non-circulatory term is dependent on the flow inertia and, hence, the airfoil lateral speed. It thus reaches its maximum around the mean angle of pitching motion and its minimum at the pitching extremes as the airfoil comes to instantaneous stops. Eventually, a like-opposite phase difference is formed between the two lift components. In addition, increasing the frequency has a negligible effect on the phase shift of the circulatory term and a slightly more pronounced impact on the phase shift of the non-circulatory term. Moreover, increasing the frequency has the role of reducing the values of the circulatory components and increasing those of the non-circulatory components. However, the impact of frequency increase is much more pronounced in the noncirculatory component. As the frequency is doubled, the non-circulatory is doubled whereas the circulatory component decreases by about $10 \%$. This aspect may demonstrate the dominance of the apparent mass effect at high reduced frequencies. The combination between the circulatory response and apparent mass yields the flow response at different reduced frequencies demonstrated earlier. 


\section{Conclusions}

The present study has been devoted to investigate the impact of mean angle of attack and frequency on the temporal variation of aerodynamic coefficients of a pitching NACA0009 airfoil. Numerical simulation results have been compared with those of theoretical analysis and showed good agreement. The structure of the flowfield around the airfoil at the different operating conditions has been explored. Better understanding of the nature of lift generation has been achieved based on the theoretical approach results.

\section{References}

[1] AGARD Report No.702, Compendium of Unsteady Aerodynamic Measurements, 1982.

[2] Theodorsen, T. "NACA Report No. 496, General Theory of Aerodynamic Instability and the Mechanism of Flutter," 1949.

[3] Chiang, H. D. and Fleeter, S., "Prediction of Incidence Effects on Oscillating Airfoil Aerodynamics by a Locally Analytical Method," International Journal for Numerical Methods in Engineering, Vol. 26, Issue 10, 1988, pp. 2227-2238.

[4] J. A. Ekaterinaris, J. A. and Menter, F. R., "Computation of Oscillating Airfoil Flows with One and Two Equation Turbulence Models," AIAA Journal Vol. 32, No. 12, 1994, pp. 2359-2365.

[5] Ko, S. and McCroskey, W. J., "Computations of Unsteady Separating Flows over an Oscillating Airfoil,” AIAA Journal, Vol. 35, No. 7, 1997, pp. 1235-1238.

[6] Srinivasan, G. R., Ekaterinaris, J. A., and McCroskey, W. J., "Evaluation of Turbulence Models for Unsteady Flows of an Oscillating Airfoil," Computers and Fluids, Vol. 24, No. 7, 1995, pp. 833-861.

[7] Ahmadi, S. A., Sharif, S., and Jamshidi, R., 'A Numerical Investigation on the Dynamic Stall of a Wind Turbine Section Using Different Turbulent Models," World Academy of Science, Engineering and Technology, Vol. 3, No. 10, 2009, pp. 292--298.

[8] Alam, M. F., Suzen, Y. B., and Ol. M. V., "Numerical Simulations of Pitching Airfoil Flowfields for MAV Applications," AIAA paper 2009-4029, 39th AIAA Fluid Dynamics Conference 22 - 25 June 2009, San Antonio, Texas,

[9] Kumar, R. and Singh, N., "Unsteady Transonic Aerodynamic Analysis for Oscillatory Airfoils and Oscillating Flaps Using Dynamic Mesh“.

[10] Wang, S., Ingham, D. B., Ma, L., Pourkashanian, M., and Tao, Z., "Numerical investigations on dynamic stall of low Reynolds number flow around oscillating airfoils," Computers and Fluids, Vol. 39, Issue 9, 2010, pp. 1529-1541.

[11] Ghoreyshi, M. and Cummings, R. M., "Challenges in the Aerodynamics Modeling of an Oscillating and Translating Airfoil at Large Incidence Angles," Aerospace Science and Technology, Vol. 28, Issue 1, 2013, pp. 176-190.

[12] Amiralaei, M. R., Alighanbari, H. and Hashemi, S. M., "An Investigation of the Effects of Unsteady Parameters on the Aerodynamics of a Low Reynolds Number Pitching Airfoil," Journal of Fluid and Structures, Vol. 26, Issue 6, 2010, pp 979-993.

[13] Lu, K., Xie, Y. H., Zhang, D., and Lan, J. B., "Numerical investigations into the asymmetric effects on the aerodynamic response of a pitching airfoil," Journal of Fluids and Structures, Vol. 39, 2013, pp. 76-86.

[14] Gülçat, Ü. "Fundamentals of Modern Unsteady Aerodynamics, Second Edition" Springer, ISBN 978-981-10-0016-4, 2015.

[15] Goett, H. J. and Bullivant, W. K., "Tests of NACA 0009, 0012 and 0018 Airfoils in the Full-Scale Tunnel," NACA Report 647, 1939.

[16] ANSYS Fluent 14.5.7 User Guide.

[17] Tolouei, E., Mani, M., Soltani, M. R., and Boroomand, M. "Analysis around a Pitching Airfoil," AIAA paper 2004-5200, 22nd Applied Aerodynamics Conference and Exhibit 16 - 19 August 2004, Providence, Rhode Island. 